\title{
Relationship between Age and Neurogenesis in Old World Monkeys
}

\author{
Tarique D. Perera ${ }^{1 *}$, Arkadiy Yaretskiy ${ }^{2}$,Anna V. Rozenboym ${ }^{3}$, Zeena Audi ${ }^{1}$, Cecilia Lipira ${ }^{1}$, \\ Jean Tang ${ }^{1}$, Jeremy Hill ${ }^{1}$, Lakshmi Thirumangalakudi ${ }^{1}$, Daniel C. Lee ${ }^{4}$, Andrew J. Dwork ${ }^{1,5}$, \\ Jeremy D. Coplan ${ }^{2}$
}

\begin{abstract}
${ }^{1}$ Departments of Psychiatry, College of Physicians and Surgeons, Columbia University Medical Center and New York State Psychiatric Institute, New York, USA; ${ }^{2}$ Nonhuman Primate Facility, Department of Psychiatry, SUNY Downstate Medical Center, Brooklyn, USA; ${ }^{3}$ Department of Biological Sciences, Kingsborough Community College, Brooklyn, USA; ${ }^{4}$ Division of Cardiothoracic Surgery, SUNY Downstate Medical Center, Brooklyn, USA; ${ }^{5}$ Department of Molecular Imaging and Neuropathology, New York State Psychiatric Institute, New York, USA.
\end{abstract}

Email: ${ }^{*}$ tp119@columbia.edu, tariqueperera@gmail.com

Received February $12^{\text {th }}, 2013$; revised March $11^{\text {th }}, 2013$; accepted April $14^{\text {th }}, 2013$

Copyright (C) 2013 Tarique D. Perera et al. This is an open access article distributed under the Creative Commons Attribution License, which permits unrestricted use, distribution, and reproduction in any medium, provided the original work is properly cited.

\begin{abstract}
Hippocampal neurogenesis continues throughout the lifespan of adult mammals, but the rates decline dramatically with increasing age. Among the factors that have been shown to affect neurogenesis, aging has been shown to be one of its most potent regulators in mice. The mechanism for the decline in neurogenesis with age is thought to be related to age-dependent changes in local and systemic neuroendocrinology and neurochemistry, as well as internal changes to precursor cells that result in decreased reactivity to normal stimuli. Since most of the data about neurogenesis and age were established from rodent studies, we sought to study this relationship in nonhuman primates in five previously studied cohorts of bonnet monkeys (Macaca radiata). In the present study, we statistically analyze the relationship of age and hippocampal neurogenesis rates, as measured by the number of DCX expressing cells in the subgranular zone of the dentate gyrus in 71 subjects with ages ranging from 3.5 to 17 years. We observed a non-significant relationship between age and doublecortin for subjects less than nine years old (corresponding to young and full adulthood) but a linear significant decline for subjects 9 years or greater (middle age and senescence). In contrast to previous studies that show neurogenesis to decline linearly throughout the lifespan, this study shows that neurogenesis occurs steadily throughout adulthood and begins to decline in middle age in bonnet macaques.
\end{abstract}

Keywords: Neurogenesis; Age; Macaca Radiate; Nonhuman Primates

\section{Introduction}

Neurogenesis, the birth of new neurons, occurs throughout adulthood in the dentate gyrus of the hippocampus [1]. The process of hippocampal neurogenesis can be divided into several developmental steps, beginning with the proliferation of precursor cells, their survival and migration, and finally, their differentiation into mature functional neurons. Newborn precursor cells arise from the subgranular zone of the dentate gyrus and, once having matured into granule cell neurons, and extend axonal projections along the mossy fiber tract to the $\mathrm{CA} 3$ region of the hippocampus, becoming integrated into the hippocampal circuitry [2-5].

${ }^{*}$ Corresponding author.
With increasing age, there is a continuous decline in precursor cell proliferation and net hippocampal neurongenesis that occurs in rat [6] and mouse models [7]. More recent experimental designs using primates showed similar results. In New World marmoset nonhuman primates, the number of bromodeoxyuridine (BrdU)-labeled cells in the dentate gyrus was shown to decline linearly with age [8]. To further study the relationship of neurogenesis and age in primates, we performed a correlation analysis on data collected from 71 adult bonnet monkeys obtained under the rubric of five separate studies: the "Fluoxetine" study, the "ECS" study, the "Ziprasidone" study and two separate "Variable Foraging Demand" (VFD) studies (see Table 1). In contrast to earlier experiments conducted in New World monkeys by other 
Table 1. Summary of five different nonhuman primate studies.

\author{
"Fluoxetine" Study \\ "Variable Foraging Demand" (VFD) \\ Study 1 and Study \\ “Electroconvulsive Shock”( ECS) Study \\ "Ziprasidone" study
}

Study of the effects of fluoxetine treatment on neurogenesis in normally reared primates and subjects exposed to separation stress conditions [13].

VFD-reared and normally reared adolescent bonnet macaques were exposed to a mild fear-provoking stimulus 2 years after the end of differential rearing in order to examine the nature and persistence of VFD rearing.

(Coplan, Andrews et al. [14]; Rosenblum et al. [15]).

Examination of whether neurogenesis is increased following electroconvulsive shock treatment [47].

Effects of Ziprasidone on neurogenesis [16]. investigators [8,9], our analysis contains significantly more subjects. Furthermore, we utilize the bonnet macaque, an Old World monkey, which possesses greater phylogenetic proximity to humans than the New World monkeys from previous studies. The two lines are believed to have split from each other approximately 35 million years ago [10].

The bonnet macaque (Macaca radiata) is an Old World monkey that reaches sexual maturity at $5-6$ years of age for males, and after about 3 years of age \pm 4 months for females $[11,12]$. From our experience in our colony, senescence in bonnet monkeys begins at around twelve to fourteen years. Here, we statistically analyze the relationship of age and neurogenesis, as reflected by the number of doublecortin (DCX) expressing cells, in the subgranular zone of the dentate gyrus in subjects ranging from 3.5 to 17 years of age.

\section{Methods}

\subsection{Fluoxetine Study}

Socially housed adult, female bonnet macaque monkeys were randomized into five groups: Control-Placebo $(\mathrm{n}=$ $3)$, Control-Drug $(\mathrm{n}=3)$, Stress-Placebo $(\mathrm{n}=3)$, Stress-Drug $(n=5)$, and Radiation-Stress-Drug $(n=4)$. The Control groups were exposed to non-stressful ambient conditions for 15 weeks, while the Stress groups were exposed to chronic stress involving social isolation for 2 days a week and home-cage reunion for 5 days a week for a total of 15 weeks. During this period, half of the controls (Control-Drug) and half of the stressed subjects (Stress-Drug) were administered fluoxetine in its once weekly preparation (Prozac Weekly from Eli Lilly Co.) at a dose of $13.5 \mathrm{mg} / \mathrm{kg} /$ week via nasogastric tubing (NGT), while the remaining animals (Control-Placebo and Stress-Placebo) received saline placebo also via NGT once a week for 15 weeks. Both treatments were administered under ketamine/xylazine sedation. The Radiated-Stress-Drug (XRT) group received bilateral temporal lobe gamma-irradiation $(20$ or 30 Gy fractionated over 10 sessions) under ketamine/xylazine sedation daily for two weeks. Following two weeks of rest, the XRT group was exposed to concurrent stress and drug administration (identical to the Stress-Drug group) for 15 weeks. All five groups were sacrificed using perfusion methods on week 15 [13].

\subsection{ECS Study}

Single-housed adult male bonnet monkeys were randomized to two groups. The ECS group $(n=6)$ received 12 administrations of high dose $(350 \%$ above seizure threshold) brief-pulse $(1.5 \mathrm{msec})$ bilateral ECS under general anesthesia (methohexital) and muscle relaxation (succinylcholine) over a 4-week period. The SHAM group (n $=6$ ) only received anesthesia and muscle relaxation over this 4-week period. Four ECS, and 4 SHAM subjects were transcardially perfused immediately following the study (Immediate-sacrifice group) while 2 ECS and 2 SHAM were perfused 4 weeks after the completion of treatment (Delayed-sacrifice group).

\subsection{Variable Foraging Demand (VFD) Study}

Single-housed adult male bonnet macaques that were exposed to maternal variable foraging demand (VFD group) or normal rearing [low foraging demand (LFD group)] during infancy were sacrificed in order to examine neurogenesis rates. The VFD group consisted of adult bonnets that were subjected to VFD for four months during infancy (first year of life). Rearing stress stemmed from maternal uncertainty when mother-infant dyads were exposed to Variable Foraging Demand (VFD). During this period, the LFD group was exposed to normal rearing conditions stemming from non-stressful foraging conditions. Previous studies have shown that VFD-reared offspring manifest behavioral and biological abnormalities reflective of mood and anxiety disorders that persist into adulthood $[14,15]$.

\subsection{Ziprasidone Study}

Single-housed female adult bonnet macaques were randomized into two cohorts. The first cohort $(\mathrm{n}=12)$ con- 
sisted of Stress-Drug $(\mathrm{n}=3)$, Stress-Placebo $(\mathrm{n}=3)$, Control-Drug $(\mathrm{n}=3)$ and Control-Placebo $(\mathrm{n}=3)$. In the second cohort $(\mathrm{n}=10)$, all subjects were exposed to stressful conditions. The Drug group $(\mathrm{n}=5)$ received ziprasidone whereas the control received placebo. The control groups were exposed to non-stressful ambient conditions while the stress group was exposed to repeated separation stress. The drug groups received ziprasidone $4.5 \mathrm{mg} / \mathrm{kg}$ daily five times/week [16].

\subsection{Immunohistology}

In all studies, subjects were sacrificed by transcardiac saline perfusions and the brains were immediately extracted and post-fixed. The left hippocampus was sectioned into $40 \mu \mathrm{m}$ free floating slices using a freezing microtome and stored in 40 wells with cryoprotectant and sodium azide $(\mathrm{NaN} 3)$ at $20^{\circ} \mathrm{C}$. These sections were immuno-labeled to detect neurogenesis and different stages of neuronal maturation. Immature neurons were indicated by the expression of doublecortin (DCX). The age of DCX-expressing neurons is based on data generated in rodent studies [17]. In nonhuman primates, DCXexpressing cells with analogous morphology seem to be 2 - 3 fold older [18-20]. For example, DCX-expressing neurons with mature dendritic morphology (stage 3) may be $8-12$ weeks old in the monkey.

Raters blinded to treatment condition counted all DCX-expressing cells in the subgranular zone (SGZ) of the dentate gyrus. To enable counting of cell clusters, the cells are examined at $\times 100$ magnification under oil immersion, omitting cells in the outermost focal plane. The densities of labeled cells are calculated by dividing the total number of cells counted by the volume of SGZ, at that level (outlined area $\times 40 \mu \mathrm{m}$ ). In order to generate true density, we divided the total number of cells identified by the total volume examined. The total number of stained cells, in contrast to the density, is relatively impervious to the effects of swelling or shrinkage of tissues. In order to determine the total number of cells identified, we employed systematic uniform random sampling, so that all rostrocaudal levels have an equal chance of being counted and assuring that no tissue was lost.

\subsection{Statistical Analyses}

The first step was to generate means and standard deviations of the five different studies that were used for the examination of the relationship between age and neurogenesis for neurogenesis rates, age and body weight. The studies were compared using a one-way ANOVA and compared individually using post-hoc Newman-Keuls testing. Sex distribution for the overall study was assessed using a Chi-square analysis. Significant effects were to be used as a control variable in subsequent gen- eral linear models. Because each of the five studies were performed within a single sex, there was a confound between study and sex. Thus, sex could not be used as a factor when the study was used and separate general linear models were implemented. A final factor, coding whether or not a subject was an experimental control, was used for the general linear models. This factor was used to control for any potential bias introduced by effects on neurogenesis induced by experimental manipulation.

For continuous measures analyses, univariate correlations were performed using Pearson's Correlation Coefficient regressing age versus neurogenesis and weight versus neurogenesis. To follow up the latter analysis, a Pearson's Correlation Coefficient was performed only in the control subjects to demonstrate that the relationship between age and neurogenesis was not dependent on potential bias introduced by experimental subjects.

A general linear model was then employed using doublecortin (DCX) labeling as the dependent variable, entering study and experimental status (control versus non-control) as categorical variables, and age and weight as continuous predictor variables. Sex could not be examined in this model as it was confounded by study. Therefore, a second general linear model was performed where study number was omitted as a variable and sex was included as a categorical variable.

For categorical measures analyses, the subjects were split into two age groups with the median age of $<9$ years of age as the "younger" group and 9 years or greater for the "older" group. The median split would correspond to middle adulthood in humans. The two groups were compared by t-tests and then Pearson's correlations were performed in each group separately.

Probability values were deemed significant at $p \leq 0.05$, two-tailed. Standard deviation was taken into account throughout all analyses.

\section{Results}

As a first step, we examined the means and standard deviations of the doublecortin staining rates for the five different studies that were used for the examination of the relationship between age and neurogenesis. As can be observed in Table 2, there is a marked difference between studies $[\mathrm{F}(4 ; 67)=4.24 ; \mathrm{p}=0.004]$.

On post-hoc Newman-Keuls analysis, it was revealed that the "ziprasidone" group exhibited neurogenesis rates that were significantly reduced in comparison to the "ECS" group and the "VFD1" group $(p<0.05)$. "Study" was therefore used as a covariate for general linear analyses.

A similar significant effect was noted for age in that the ziprasidone study exhibited increases in age versus the remaining subjects (Post-hoc Newman-Keuls: p < 
Table 2. Means and standard deviations for five different nonhuman primate studies on neurogenesis, age, and weight.

\begin{tabular}{|c|c|c|c|}
\hline Study-Means \pm Sd & Doublecortin $^{1}$ & ${ }^{*}$ Age $^{2}-$ & Weight $^{3}$ - \\
\hline “Fluoxetine" (N = 18) & $97.2 \pm 76.2$ & $9.7 \pm 3.0$ & $5.1 \pm 1.4$ \\
\hline “ECS" $(\mathbf{N}=8)^{*}$ & $136.6 \pm 138$ & $7.3 \pm 0.5$ & $7.91 \pm 0.9$ \\
\hline “Ziprasidone” $(\mathrm{N}=21)$ & $20.1 \pm 26.9$ & $13.6 \pm 2.1$ & $5.8 \pm 1.2$ \\
\hline “VFD1" $(\mathrm{N}=8)$ & $114.1 \pm 93.3$ & $8.8 \pm 1.8$ & $10.4 \pm 2.9$ \\
\hline “VFD2” (N = 17) & $68 \pm 96.3$ & $9.6 \pm 2.5$ & $7.5 \pm 3.3$ \\
\hline All Grps (N = 72) & $74.1 \pm 89.7$ & $10.5 \pm 3.1$ & $6.8 \pm 2.6$ \\
\hline
\end{tabular}

$\overline{1}$. Overall group difference using one-way ANOVA $[\mathrm{F}(4 ; 67)=4.24 ; \mathrm{p}=0.004] .{ }^{2}$. Overall group difference using one-way ANOVA $[\mathrm{F}(4 ; 66)=15.13 ; \mathrm{p}<$ $0.0001] .{ }^{3}$. Overall group difference for weight using one-way ANOVA $[\mathrm{F}(4 ; 67)=11.28 ; \mathrm{p}<0.0001] .{ }^{*}$ one subject is missing for age for ECS study.

0.01 for all comparisons). None of the other groups revealed significant comparisons between-groups. This again was controlled for by using "study" as a control variable.

Moreover, a significant weight effect was observed. On Newman-Keuls post-hoc testing, significant differences $(\mathrm{p}<0.05)$ are noted between all study groups except between the "fluoxetine" and "ziprasidone" studies and between the "ECS" and "VFD2" study. Thus, "weight" was used as a covariate.

For sex distribution, 33 subjects were male whereas 37 were female. No difference in sex distribution was noted, but because of potential sex differences, sex was used as a factor or covariate, depending on the analysis.

\subsection{Continuous Measures}

For univariate Pearson's correlations, weight did not correlate significantly with neurogenesis rates as reflected by doublecortin $(\mathrm{r}=-0.10 ; \mathrm{N}=72 ; \mathrm{p}=0.39)$ whereas age did correlate significantly with neurogenesis rates $(\mathrm{r}=-0.47 ; \mathrm{N}=71 ; \mathrm{p}<0.001)$ (see Figure 1). Given the non-linear relationship between age and neurogenesis rates, we examined this relationship using univariate non-linear regression analysis, which revealed a numerically more robust relationship $[\mathrm{F}(1 ; 69)=19.88 ; \mathrm{p}$ $=0.00003]$ in comparison to the linear model (see Figure 1).

The first general linear model used doublecortin as the dependent variable, and entered study and experimental status (control versus non-control) as categorical variables, and age and weight as continuous predictor variables. Age predicted doublecortin expression (see Table 3), independent of all the aforementioned variables. As mentioned, sex is confounded with study, so sex had to be included into an additional analysis. There is a trend for study effect which in all likelihood represented lower doublecortin expression in the ziprasidone study.

We then conducted the analysis without study number, in order to examine for potential sex effects. No sex effect was observed, although a highly significant age ef-

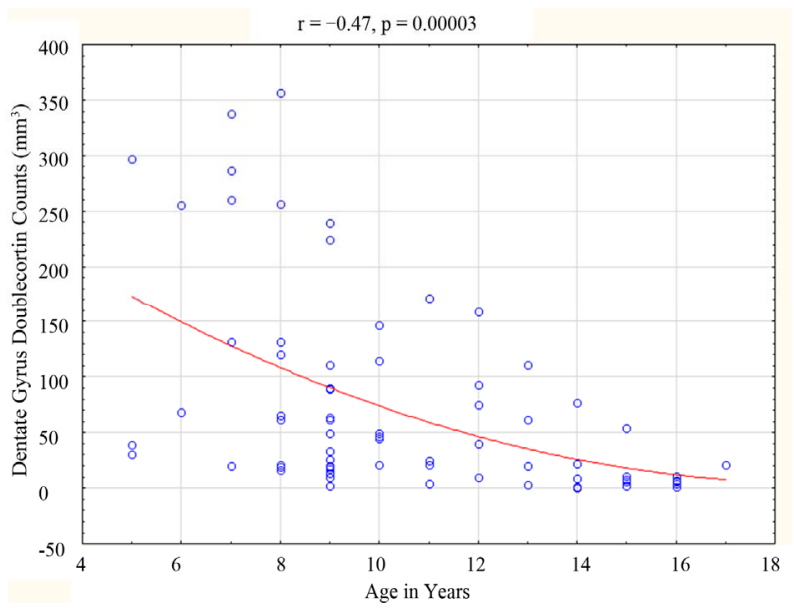

Figure 1. Scatterplot of Neurogenesis Rates of Old World Monkeys as Reflected by Rates of Doublecortin counts in the dentate gyrus of the hippocampus versus age in months.

Table 3. Prediction of doublecortin staining within the dentate gyrus of the hippocampus by age and other control variables.

\begin{tabular}{cccc}
\hline Parameter & DF & F & p \\
\hline Weight & 1 & 1.09 & 0.30 \\
Age & 1 & 18.84 & $\mathbf{0 . 0 0 0 0 5}$ \\
Study & 1 & 0.52 & 0.47 \\
Experimental Control & 1 & 0.39 & 0.53 \\
Error & 66 & & \\
\hline
\end{tabular}

fect was still evident when controlling for sex (Table 4). In this second analysis, a trend for a weight effect was also observed and is consistent with our previous paper examining correlation between weight and neurogenesis in the subjects in the "VFD1 study" [21]. A Pearson correlation run only in the control subjects revealed a significant inverse relationship between age and neurogenesis reflected as doublecortin staining $[\mathrm{r}=-0.57 ; \mathrm{N}=18$; $\mathrm{p}=0.014]$. Therefore, we demonstrate that age signifi- 
cantly predicts doublecortin expression, independent of weight, sex, study type and experimental control status.

\subsection{Categorical Measures}

When the subjects were split into two age groups with the median age of $<9$ years of age as the "younger" group and 9 years or greater for the "older" group, the younger group showed significantly more cells expressing doublecortin in comparison to the older group [139.55 \pm 122.86 in younger subjects versus $49.43 \pm 57.42(\mathrm{~N}=51)$ in older subjects; (t-value $=4.22 ; \mathrm{df}=69 ; \mathrm{p}=0.00007)$ ] (Figure 2). Correlation analyses in each group considered separately reveals no age effect in the younger group but a significant inverse age effect is observed in the older age group $(\mathrm{r}=-0.38 ; \mathrm{N}=51 ; \mathrm{p}=0.005)$. These data suggest a linear fall off of neurogenesis following full adulthood but less of an age effect during young adulthood.

\section{Discussion}

The results of this study show that with advancing age, there is a decline in neurogenesis within the subgranular

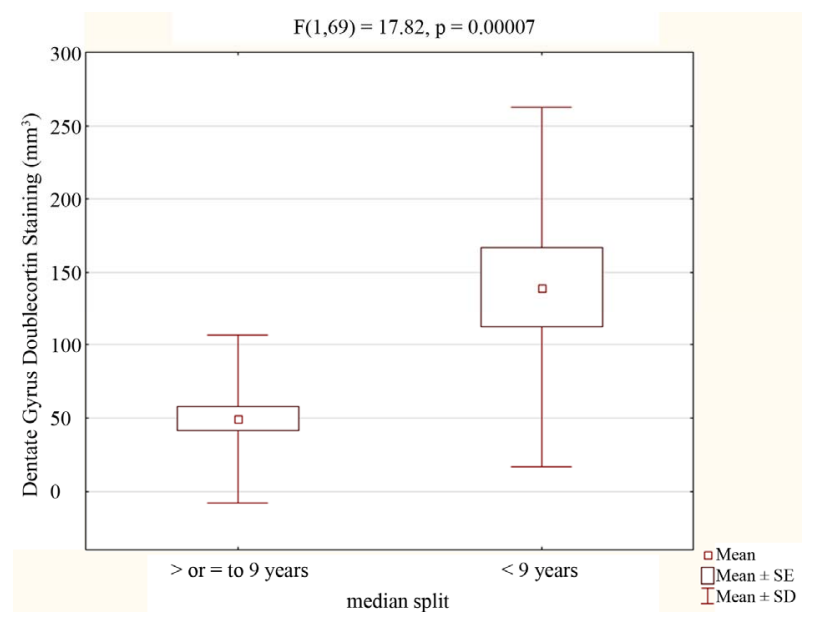

Figure 2. Mean \pm SD of neurogenesis rates of Old World monkeys as reflected by rates of doublecortin counts in the dentate gyrus of the hippocampus in subjects split based on median age.

Table 4. Prediction of doublecortin staining within the dentate gyrus of the hippocampus by age with study omitted as control variable.

\begin{tabular}{cccc}
\hline & DF & F & P \\
\hline Sex & 1 & 1.10 & 0.30 \\
Experimental Control & 1 & 0.30 & 0.58 \\
Weight & 1 & 3.16 & 0.08 \\
Age & 1 & 10.83 & $\mathbf{0 . 0 0 1 6}$ \\
Error & 66 & & \\
\hline
\end{tabular}

zone of the hippocampus in adult mammals. Our study is consistent with the results of earlier smaller studies of New World Monkeys that show an inverse relationship between neurogenesis within dentate gyrus and age [8]. However, we found that instead of a linear decline in neurogenesis throughout the bonnet macaque lifespan, we observed a non-significant relationship between age and new hippocampal neurons expressing doublecortin for subjects less than 9 years old (corresponding to young and full adulthood). However, there was a linear significant decline for subjects 9 years or greater (middle age and senescence). This suggests that neurogenesis continues steadily in the Bonnet macaque throughout lifespan until middle age and then begins to decline through middle age to senescence. The reason for the difference between our study and other studies that indicates a linear decline across the full age range [8] is unclear but may involve species differences and phylum (Old World versus New World monkeys) and the former being more closely related to humans.

The trend of weight effect that was observed when controlling for sex was consistent with our previous paper examining correlation between weight and neurogenesis in the subjects in the "VFD1 study" [21]. Statistical analysis revealed that there was an overlap between the effect of study and age. This limitation in the results was partially due to the variation of the mean ages between each study, and was especially prominent with the subjects of the ziprasidone (Geodon) study. However, we tried to minimize this effect by controlling for "study" as one of our covariates. Furthermore, excluding the ziprasidone study data from the analysis did not yield a significant difference.

\section{Mechanisms of Age Related Decline in Neurogenesis}

Aging induces important changes in neuroendocrinology and neurochemistry of the brain that involve the synthesis of neurotransmitters, growth factors, neuropeptides and steroids [22]. Age-related decline in neurogenesis can be explained by such aforementioned changes in local and systemic environments that no longer provide the necessary mitotic stimuli for cell proliferation. Previous studies show that inducers of neurogenesis decrease with age, whereas signals that interfere with neurogenesis increase with age. Indeed, age-related decreases in neurogenesis can be prevented by the deactivation of these positive regulators [23-25]. This effect is thought to occur by an increase in the number of cells within the dentate gyrus, rather than the deceleration of a slowed cell cycle. The additional dividing cells could come from precursors that have become quiescent over time, i.e., cells that are not dividing but have retained their capacity to divide [26]. 
Glucocorticoids have been shown to be among the most potent negative regulators of neurogenesis. Glucocorticoids are released into the blood circulation following the activation of the hypothalamo-pituitary-adrenal (HPA) axis, primarily by stress. Acute [27] or chronic [28] treatment with corticosterone, the main glucocorticoid in rodents, has been shown to be a strong negative regulator of cell proliferation in the adult dentate gyrus. Corticosterone-mediated downregulation of cell proliferation in the adult dentate gyrus has also been accomplished by exposing mice $[29,30]$ to various paradigms of stress. Removal of the stress stimulus, however, can completely reverse the changes in neuronal differentiation in 3 weeks [30]. Furthermore, in adult rodents, adrenalectomy stimulates cell proliferation in the dentate gyrus [31].

Chronically elevated glucocorticoid levels, therefore, are thought to be responsible, in part, for reduced neurogenesis in the aging dentate gyrus. Evidence for this comes from rodent studies that show elevated basal levels of corticosterone mainly during the dark phase of the circadian cycle, and prolonged secretion after stress. Both basal and stress-induced corticosterone secretion is blocked by an adrenalectomy in mid-adulthood, and is associated with increased cell proliferation and neurogenesis in senescent animals [32]. When adrenalectomy is performed on senescent rats, there is a decrease in corticosterone levels and increased cell proliferation in the dentate gyrus $[23,33]$. There are also changes in the response to corticosterone with age. Aged rats have higher expression of glucocorticoid receptors in early precursors, and calretinin-positive immature neurons, which are devoid of glucocorticoid receptors or mineralocorticoid receptor expression in younger mice, come to acquire both receptors in old age [34]. Thus, corticosteroid mediated decline in neurogenesis in older ages may operate via increased basal levels, as well as increased sensitivity to hormone action.

Numerous neurotransmitters have also been shown to modulate neurogenesis. Glutamate is a negative regulator of neurogenesis [35,36]. NMDA receptor antagonism can reverse the decline of neurogenesis in the aged brain [37]. Furthermore, it was shown that the effects of corticosterone on cell division could be blocked by treatments that activate or inactivate NMDA receptors suggesting that glutamate works via a common pathway with corticosteroids [38]. GABA was also shown to be a negative regulator of neurogenesis. In aged animals, infusion of Preg-S, a negative allosteric regulator of GABA binding, considerably increases the rate of cell proliferation and neurogenesis [39]. GABAergic neurotransmission, moreover, becomes elevated with age [40]. Taken together, this evidence suggests that decreases in GABA may potentially contribute to age-related decline in neurogene- sis.

Lastly, serotonin has been shown to modulate neurogenesis, mainly through the action of the presynaptic and postsynaptic $5 \mathrm{HT}_{1 \mathrm{a}}$ [41]. Studies that show decreased serotonin levels, as well as 5HT1a binding declines with ageing, supporting the idea that decreased serotonergic activity also contributes to the decline in neurogenesis with age. In addition to hormones and neurotransmitters, numerous neurotrophic factors including EGF, IGF-1, VEGF, FGF-2, and BDNF have been shown to decrease in middle age and have been associated with decreased cell proliferation in the dentate gyrus and the subventricular zone [42-45].

The studies conducted thus far suggest that multiple neuropeptides and other mediators exert a deleterious effect on neurogenesis in the hippocampus. However, it is also possible that with age, changes occur to the precursor cells themselves, such that they no longer can respond to the normal growth stimuli. While current data do suggest that the main change in precursor cells that occurs with age is their decrease in rate of proliferation, the mechanisms responsible for this effect are unclear. Recent studies using stem cell marker SOX-2 to differentiate stem cells from Brdu-labeled proliferating cells show that the overall number of Sox-2 labeled cells remains constant throughout the lifespan while the number of cells expressing endogenous proliferation marker declines significantly with age $[44,46]$. The results of this study suggest that the decline in neurogenesis rates is due to the increased quiescence of active precursor cells, rather than the progressive loss of the total number of precursors. A further comparison of the Sox-2/BrdU data with Sox-2/Ki67 in this study suggests that there is a lengthening in cell cycle that occurs with age. Thus, with age, both the increased quiescence, as well as lengthening of the cell cycle may contribute to the decline in the rate of neurogenesis.

\section{Acknowledgements}

We wish to thank Drs Christopher Lange and David Lee for their invaluable contributions

\section{REFERENCES}

[1] J. Altman and G. D. Das, "Autoradiographic and Histological Evidence of Postnatal Hippocampal Neurogenesis in Rats," The Journal of Comparative Neurology, Vol. 124, No. 3, 1965, pp. 319-335. doi: $10.1002 /$ cne. 901240303

[2] N. B. Hastings and E. Gould, "Rapid Extension of Axons into the CA3 Region by Adult-Generated Granule Cells," Journal of Comparative Neurology, Vol. 415, No. 1, 1999, pp. 144-144. doi:10.1002/(SICI)1096-9861(19991206)415:1<144::AID -CNE10>3.0.CO;2-7 
[3] E. A. Markakis and F. H. Gage, "Adult-Generated Neurons in the Dentate Gyrus Send Axonal Projections to Field CA3 and Are Surrounded by Synaptic Vesicles," The Journal of Comparative Neurology, Vol. 406, No. 4, 1999, pp 449-460. doi:10.1002/(SICI)1096-9861(19990419)406:4<449::AID -CNE3>3.0.CO;2-I

[4] H. van Praag, A. F. Schinder, B. R. Christie, N. Toni, T. D. Palmer and F. H. Gage, "Functional Neurogenesis in the Adult Hippocampus," Nature, Vol. 415, No. 6875, 2002, pp. 1030-1034. doi:10.1038/4151030a

[5] P. Ambrogini, D. Lattanzi, S. Ciuffoli, D. Agostini, L. Bertini, V. Stocchi, S. Santi and R. Cuppini, "MorphoFunctional Characterization of Neuronal Cells at Different Stages of Maturation in Granule Cell Layer of Adult Rat Dentate Gyrus," Brain Research, Vol. 1017, No. 1-2, 2004, pp. 21-31. doi:10.1016/j.brainres.2004.05.039

[6] H. G. Kuhn, H. Dickinson-Anson and F. H. Gage, "Neurogenesis in the Dentate Gyrus of the Adult Rat: Age-Related Decrease of Neuronal Progenitor Proliferation," The Journal of Neuroscience, Vol. 16, No. 6, 1996, pp. 20272033.

[7] G. Kempermann, H. G. Kuhn and F. Gage, "ExperienceInduced Neurogenesis in the Senescent Dentate Gyrus," The Journal of Neuroscience, Vol. 18, No. 9, 1998, pp. 3206-3212.

[8] B. Leuner, Y. Kozorovitskiy, C. G. Gross and E. Gould, "Diminished Adult Neurogenesis in the Marmoset Brain Precedes Old Age," Proceedings of the National Academy of Sciences, Vol. 104, No. 43, 2007, pp. 17169-17173. doi:10.1073/pnas.0708228104

[9] E. Gould, A. J. Reeves, M. Fallah, P. Tanapat, C. G. Gross and E. Fuchs, "Hippocampal Neurogenesis in Adult Old World Primates," Proceedings of the National Academy of Sciences, Vol. 96, No. 9, 1999, pp. 5263-5267. doi:10.1073/pnas.96.9.5263

[10] C. G. Schrago and C. A. M. Russo, "Timing the Origin of New World Monkeys," Molecular Biology and Evolution, Vol. 20, No. 10, 2003, pp.1620-1625. doi: $10.1093 / \mathrm{molbev} / \mathrm{msg} 172$

[11] G. R. Aravindan, N. Ravindranath, K. Gopalakrishnan and N. R. Moudgal, "DNA Flow-Cytometric Analysis of Testicular Germ Cell Populations of the Bonnet Monkey (Macaca radiata) as a Function of Sexual Maturity," Journal of Reproduction and Fertility, Vol. 89, No. 2, 1990, pp. 397-406. doi:10.1530/jrf.0.0890397

[12] A. J. Rao, V. Ramesh, S. G. Ramachandra, H. N. Krishnamurthy, N. Ravindranath and N. R. Moudgal, "Growth and Reproductive Parameters of Bonnet Monkey (Macaca radiata)," Primates, Vol. 39, No. 1, 1998, pp. 97-107. doi:10.1007/BF02557748

[13] T. D. Perera, A. J. Dwork, K. A. Keegan, L. Thirumangalakudi, C. M. Lipira, N. Joyce, C. Lange, J. D. Higley, G. Rosoklija, R. Hen, H. A. Sackeim and J. D. Coplan, "Necessity of Hippocampal Neurogenesis for the Therapeutic Action of Antidepressants in Adult Nonhuman Primates," PLoS ONE, Vol. 6, No. 4, 2011, p. 17600. doi:10.1371/journal.pone.0017600

[14] J. D. Coplan, M. W. Andrews, L. A. Rosenblum, M. J.
Owens, S. Friedman, J. M. Gorman and C. B. Nemeroff, "Persistent Elevations of Cerebrospinal Fluid Concentrations of Corticotropin-Releasing Factor in Adult Nonhuman Primates Exposed to Early-Life Stressors: Implications for the Pathophysiology of Mood and Anxiety Disorders," Proceedings of the National Academy of Sciences, Vol. 93, No. 4, 1996, pp. 1619-1623.

doi:10.1073/pnas.93.4.1619

[15] L. A. Rosenblum, C. Forger, S. Noland, R. C. Trost and J. D. Coplan, "Response of Adolescent Bonnet Macaques to an Acute Fear Stimulus as a Function of Early Rearing Conditions," Developmental Psychobiology, Vol. 39, No. 1, 2001, pp. 40-45. doi:10.1002/dev.1026

[16] J. Hill, J. E. Tang, et al., "Effects of Ziprasidone on Neurogenesis and Depressive Behavior in Adult Monkeys," Biological Psychiatry, Vol. 69, No. 9, 2011, p. 126S.

[17] S. Couillard-Despres, B. Winner, S. Schaubeck, R. Aigner, M. Vroemen, N. Weidner, U. Bogdahn, J. Winkler, H. G. Kuhn and L. Aigner, "Doublecortin Expression Levels in Adult Brain Reflect Neurogenesis," European Journal of Neuroscience, Vol. 21, No. 1, 2005, pp. 1-14. doi:10.1111/j.1460-9568.2004.03813.x

[18] D. R. Kornack and P. Rakic, "Continuation of Neurogenesis in the Hippocampus of the Adult Macaque Monkey," Proceedings of the National Academy of Sciences, Vol. 96, No. 10, 1999, pp. 5768-5773. doi: $10.1073 /$ pnas. 96.10 .5768

[19] L. B. Ngwenya, A. Peters and D. L. Rosene, "Maturational Sequence of Newly Generated Neurons in the Dentate Gyrus of the Young Adult Rhesus Monkey," The Journal of Comparative Neurology, Vol. 498, No. 2, 2006, pp. 204-216. doi:10.1002/cne.21045

[20] L. B. Ngwenya, D. L. Rosene and A. Peters, "An Ultrastructural Characterization of the Newly Generated Cells in the Adult Monkey Dentate Gyrus," Hippocampus, Vol. 18, No. 2, 2008, pp. 210-220. doi:10.1002/hipo.20384

[21] T. D. Perera, D. Lu, L. Thirumangalakudi, E. L. Smith, A. Yaretskiy, L. A. Rosenblum, J. G. Kral and J. D. Coplan, "Correlations between Hippocampal Neurogenesis and Metabolic Indices in Adult Nonhuman Primates," Neural Plasticity, Vol. 2011, 2011, pp. 1-6. doi: $10.1155 / 2011 / 875307$

[22] R. G. Smith, L. Betancourt and Y. Sun, "Molecular Endocrinology and Physiology of the Aging Central Nervous System," Endocrine Reviews, Vol. 26, No. 2, 2005, pp. 203-250. doi:10.1210/er.2002-0017

[23] H. A. Cameron and R. D. G. McKay, "Restoring Production of Hippocampal Neurons in Old Age," Nature Neuroscience, Vol. 2, No. 10, 1999, pp. 894-897. doi:10.1038/13197

[24] K. Jin, Y. Sun, L. Xie, S. Batteur, X. O. Mao, C. Smelick, A. Logvinova and D. A. Greenberg, "Neurogenesis and Aging: FGF-2 and HB-EGF Restore Neurogenesis in Hippocampus and Subventricular Zone of Aged Mice," Aging Cell, Vol. 2, No. 3, 2003, pp. 175-183. doi:10.1046/j.1474-9728.2003.00046.x

[25] W. P. Gray, K. May and L. E. Sundström, "Seizure Induced Dentate Neurogenesis Does Not Diminish with Age in Rats," Neuroscience Letters, Vol. 330, No. 3, 
2002, pp. 235-238 doi:10.1016/S0304-3940(02)00810-8

[26] A. Olariu, K. M. Cleaver and H. A. Cameron, "Decreased Neurogenesis in Aged Rats Results from Loss of Granule Cell Precursors without Lengthening of the Cell Cycle," The Journal of Comparative Neurology, Vol. 501, No. 4, 2007, pp. 659-667. doi:10.1002/cne.21268

[27] H. A. Cameron and E. Gould, "Adult Neurogenesis Is Regulated by Adrenal Steroids in the Dentate Gyrus," Neuroscience, Vol. 61, No. 2, 1994, pp. 203-209. doi:10.1016/0306-4522(94)90224-0

[28] P. Ambrogini, L. Orsini, C. Mancini, P. Ferri, I. Barbanti and R. Cuppini, "Persistently High Corticosterone Levels but Not Normal Circadian Fluctuations of the Hormone Affect Cell Proliferation in the Adult Rat Dentate Gyrus," Neuroendocrinology, Vol. 76, No. 6, 2002, pp. 366-372. doi:10.1159/000067581

[29] K. Pham, J. Nacher, P. R. Hof and B. S. McEwen, "Repeated Restraint Stress Suppresses Neurogenesis and Induces Biphasic PSA-NCAM Expression in the Adult Rat Dentate Gyrus," European Journal of Neuroscience, Vol. 17, No. 4, 2003, pp. 879-886. doi:10.1046/j.1460-9568.2003.02513.x

[30] V. M. Heine, S. Maslam, J. Zareno, M. Joëls and P. J. Lucassen, "Suppressed Proliferation and Apoptotic Changes in the Rat Dentate Gyrus after Acute and Chronic Stress Are Reversible," European Journal of Neuroscience, Vol. 19, No. 1, 2004, pp. 131-144. doi:10.1046/j.1460-9568.2003.03100.x

[31] E. Gould, H. A. Cameron, D. C. Daniels, C. S. Woolley and B. S. McEwen, "Adrenal Hormones Suppress Cell Division in the Adult Rat Dentate Gyrus," The Journal of Neuroscience, Vol. 12 , No. 9, 1992, pp. 3642-3650.

[32] M. F. Montaron, E. Drapeau, D. Dupret, P. Kitchener, C. Aurousseau, M. Le Moal, P. V. Piazza and D. N. Abrous, "Lifelong Corticosterone Level Determines Age-Related Decline in Neurogenesis and Memory," Neurobiology of Aging, Vol. 27, No. 4, 2006, pp. 645-654. doi:10.1016/j.neurobiolaging.2005.02.014

[33] M. F. Montaron, K. G. Petry, J. J. Rodriguez, M. Marinelli, C. Aurousseau, G. Rougon, M. Le Moal and D. N. Abrous, "Adrenalectomy Increases Neurogenesis but Not PSA-NCAM Expression in Aged Dentate Gyrus," European Journal of Neuroscience, Vol. 11, No. 4, 1999, pp. 1479-1485. doi:10.1046/j.1460-9568.1999.00579.x

[34] A. Garcia, B. Steiner, G. Kronenberg, A. Bick-Sander and G. Kempermann, "Age-Dependent Expression of Glucocorticoid- and Mineralocorticoid Receptors on Neural Precursor Cell Populations in the Adult Murine Hippocampus," Aging Cell, Vol. 3, No. 6, 2004, pp. 363-363. doi:10.1111/j.1474-9728.2004.00130.x

[35] H. A. Cameron, B. S. McEwen and E. Gould, "Regulation of Adult Neurogenesis by Excitatory Input and NMDA Receptor Activation in the Dentate Gyrus," The Journal of Neuroscience, Vol. 15, No. 6, 1995, pp. 4687-4692.

[36] J. Nacher, D. R. Rosell, G. Alonso-Losa and B. S. McEwen, "NMDA Receptor Antagonist Treatment Induces a Long-Lasting Increase in the Number of Proliferating Cells, PSA-NCAM-Immunoreactive Granule Neurons and Ra- dial Glia in the Adult Rat Dentate Gyrus," European Journal of Neuroscience, Vol. 13, No. 3, 2001, pp. 512-520. doi:10.1046/j.0953-816x.2000.01424.x

[37] J. Nacher, G. Alonso-Llosa, D. R. Rosell and B. S. McEwen, "NMDA Receptor Antagonist Treatment Increases the Production of New Neurons in the Aged Rat Hippocampus," Neurobiology of Aging, Vol. 24, No. 2, 2003, pp. 273-284.doi:10.1016/S0197-4580(02)00096-9

[38] H. A. Cameron, P. Tanapat and E. Gould, "Adrenal Steroids and N-Methyl-D-Aspartate Receptor Activation Regulate Neurogenesis in the Dentate Gyrus of Adult Rats through a Common Pathway," Neuroscience, Vol. 82, No. 2, 1997, pp. 349-354. doi:10.1016/S0306-4522(97)00303-5

[39] W. Mayo, O. George, S. Darbra, J. J Bouyer, M. Vallée, M. Darnaudéry, M. Pallarès, V. Lemaire-Mayo, M. Le Moal, P. V. Piazza and N. Abrous, "Individual Differences in Cognitive Aging: Implication of Pregnenolone Sulfate," Progress in Neurobiology, Vol. 7, No. 1, 2003, pp. 43-48. doi:10.1016/j.pneurobio.2003.09.006

[40] G. Segovia, A. G. Yagüe, J. M. García-Verdugo and F. Mora, "Environmental Enrichment Promotes Neurogenesis and Changes the Extracellular Concentrations of Glutamate and GABA in the Hippocampus of Aged Rats," Brain Research Bulletin, Vol. 70, No.1, 2006, pp. 8-14. doi:10.1016/j.brainresbull.2005.11.005

[41] M. Banasr, M. Hery, R. Printemps and A. Daszuta, "Serotonin-Induced Increases in Adult Cell Proliferation and Neurogenesis Are Mediated through Different and Common 5-HT Receptor Subtypes in the Dentate Gyrus and the Subventricular Zone," Neuropsychopharmacology, Vol. 29, No. 3, 2004, p. 11. doi:10.1038/sj.npp.1300320

[42] H. G. Kuhn, J. Winkler, G. Kempermann, L. J. Thal and F. H. Gage, "Epidermal Growth Factor and Fibroblast Growth Factor-2 Have Different Effects on Neural Progenitors in the Adult Rat Brain," The Journal of Neuroscience, Vol. 17, No. 15, 1997, pp. 5820-5829.

[43] E. Enwere, T. Shingo, C. Gregg, H. Fujikawa, S. Ohta and S. Weiss, "Aging Results in Reduced Epidermal Growth Factor Receptor Signaling, Diminished Olfactory Neurogenesis, and Deficits in Fine Olfactory Discrimination," The Journal of Neuroscience, Vol. 24, No. 38, 2004 , pp. 8354-8365. doi:10.1523/JNEUROSCI.2751-04.2004

[44] A. K. Shetty, B. Hattiangady and G. A. Shetty, "Stem/ Progenitor Cell Proliferation Factors FGF-2, IGF-1, and VEGF Exhibit Early Decline during the Course of Aging in the Hippocampus: Role of Astrocytes," Glia, Vol. 51, No. 3, 2005, pp. 173-186. doi:10.1002/glia.20187

[45] T. D. Palmer, A. R. Willhoite and F. H. Gage, "Vascular Niche for Adult Hippocampal Neurogenesis," The Journal of Comparative Neurology, Vol. 425, No. 4, 2000, pp 479-494.

doi:10.1002/1096-9861(20001002)425:4<479::AID-CNE 2>3.0.CO;2-3

[46] B. Hattiangady and A. K. Shetty, "Aging Does Not Alter the Number or Phenotype of Putative Stem/Progenitor Cells in the Neurogenic Region of the Hippocampus," Neurobiology of Aging, Vol. 29, No. 1, 2008, pp. 129-147. doi:10.1016/j.neurobiolaging.2006.09.015 
[47] T. D. Perera, J. D. Coplan, C. M. Lipira, C. Carpio, M. Arif, G. B. Rosoklija, S. Lisanby, G. B. Spitzer, B. Scharf, A. J. Dwork, L. A. Rosenblum and H. A. Sackeim, "ECS
Induces Neurogenesis in the Adult Monkey Dentate Gyrus," Journal of Neuroscience, Vol. 27, No. 18, 2007, pp. 4894-4901. 